\title{
Préface
}

\section{« Le lieu introuvable du pardon »}

\author{
Cristina de Peretti \\ Doctora en Filosofía \\ UNED Madrid (España) \\ mperetti@sof.uned.es
}

Como citar este artículo: C. PERETTI "Préface « Le lieu introuvable du pardon »" en Palabra y Razón. Revista de Teología, Filosofía y Ciencias de la Religión No15, Julio 2019, pp. 99-104 https://doi.org/10.29035/pyr.15.99

Le philosophe chilien Javier Agüero a consacré ses recherches à la psychanalyse, à la philosophie française contemporaine -avec une attention toute particulière à la pensée de Jacques Derrida- ainsi qu'à toute une série de questions qui se recoupent et qui portent, à leur tour, sur ce que l'on appelle grosso modo la «philosophie politique » : la violence, la démocratie et les droits de l'homme, l'hospitalité, la communauté et l'extranéité, la marginalité mais encore l'héritage, la mémoire, l'oubli, le pardon, etc. Et ce sont justement quelques-unes de ces problématiques groupées -comme son titre, Les Silences du pardon. Sur le Chili post-Pinochet, l'indique- autour du pardon que ce livre va reprendre afin d'engager un débat sur le processus chilien de transition et de démocratisation mis en œuvre après toutes les horreurs que ce «pays d'Antigones », comme dit Agüero, a dû souffrir sous la dictature militaire de Pinochet, après le coup d'État de 1973.

Suite à la Seconde Guerre mondiale et à l'instauration, lors du procès de Nuremberg, du concept de « crime contre l'humanité » et en réponse également à d'autres évènements historiques et politiques graves ultérieurs, depuis les dernières décennies du Xxème siècle, les déclarations publiques de repentance, d'(auto) accusation pour des fautes et des crimes passés ou présents et de demande de pardon n'ont certes pas cessé de se multiplier à l'échelle géopolitique mondiale. Mondialisation ou, comme aime à dire Derrida, « mondialatinisation »-qui « devient désormais européo-anglo-américaine dans son idiome $»^{1}$, précise-t-il- du pardon

\footnotetext{
${ }^{1}$ J. DERRIDA, « Foi et savoir. Les deux sources de la "religion” aux limites de la simple raison », in $F o i$ et Savoir, suivi de Le Siècle et le Pardon (Entretien avec Michel Wieviorka). Seuil: Paris, 2000, p. 66.
} 
dont les différentes manifestations ainsi que le vocabulaire et la rhétorique -ces derniers issus foncièrement de l'héritage abrahamique et d'un certain humanisme philosophique- marquent de leur empreinte nos sociétés contemporaines ainsi que leur langage politique et juridique internationalisé. Cependant, le pardon, rappelle souvent Derrida, «n'est pas une question politique au sens traditionnel [...] Le pardon n'appartient ni au champ du politique ni au champ du juridique. On ne peut pas inscrire le pardon dans le droit $»^{2}$ car le pardon « ne rend pas justice, c'est certain, il ne se substitue pas à la justice, la valeur du pardon est hétérogène à la valeur de jugement juridique $»^{3}$.

Cela dit, le pardon n'en est pas moins un concept complexe et équivoque, traversé d'apories, qui soulève, partant, un certain nombre de problèmes et de questions : est-ce quelque chose ou quelqu'un que l'on pardonne ? Est-ce à la victime ou à Dieu, le « témoin absolu » ${ }^{4}$ par excellence, que l'on demande pardon ? Ou est-ce encore, ces derniers temps, à cet autre témoin -sinon absolu, néanmoins collectifqu'est l'opinion publique, etc. ? Est-ce que le pardon suppose forcément un face à face singulier entre la victime et le coupable ?5 Et, si c'est le cas, est-on en droit de demander pardon ou de l'accorder au nom de l'autre ? Est-ce que le pardon peut faire l'objet d'une transaction? Est-ce que, pour être accordé, le pardon doit se conformer à des conditions de possibilité - telles que, par exemple (comme l'exige la religion chrétienne), l'aveu de la faute, le repentir, la contrition, la conversion et, partant, la rédemption du pécheur-, voire à des finalités de toute sorte : sociales, politiques, juridiques, voire psychologiques, thérapeutiques, etc. ?, etc.

C'est en partie pour répondre à ces questions ainsi qu'à maintes autres sans doute que l'auteur consacre la première partie de son livre à rappeler, d'une part, l'étymologie du mot " pardon » ainsi que ses origines religieuses, bibliques, surtout chrétiennes (comme nous venons de le constater) et, d'autre part, à rendre compte -tout en passant par Hegel (pour qui le pardon et la réconciliation sont le moteur de l'historicité)- de la façon dont Jankélévitch (dont les deux textes consacrés à ce sujet se situent l'un par rapport à l'autre dans une sorte d'oscillation dialectique) et Ricœur (qui parle de la nature anthropologique du pardon en tant qu'espace réciproque d'échange entre altérités) conçoivent le pardon.

\footnotetext{
${ }^{2}$ J. DERRIDA, «Premier entretien avec Jacques Derrida : "La solidarité des vivants". Propos recueillis par E. Nascimento», in La Solidarité des vivants et le pardon. Conférence et entretiens. Hermann: Paris, 2016, p. 136.

${ }^{3}$ J. DERRIDA, « Justice et pardon », in Sur parole. Instantanés philosophiques. Éditions de l'Aube: Paris, 1999, p. 132.

${ }^{4}$ J. DERRIDA, « Le siècle et le pardon », 113.

${ }^{5}$ J. DERRIDA, « Justice et pardon », p. 139.
} 
Toutefois, pour le difficile parcours qu'il entreprend d'effectuer, Agüero va plus particulièrement choisir deux de ses compagnons de route préférés et dont il connaît parfaitement les textes et la pensée. Je parle, bien entendu, de Derrida et de Freud... dont les héritages sans doute réciproques -psychanalyse/déconstruction mais encore déconstruction/psychanalyse : la mémoire, l'oubli ainsi que l'archive, le travail de deuil et la crypte, mais encore les restes, les cendres, les ruines et les spectres- et toujours encore à venir sont scrupuleusement analysés par l'auteur.

« [...] y a-t-il un concept de pardon, un seul $?^{6} »$, (se) demande Derrida. Et, dès l'abord, il met en garde de ne point confondre -comme c'est fréquemment le cas, parfois de propos délibéré- le pardon avec l'excuse, le repentir ou le regret, pas plus, d'ailleurs, qu'avec l'oubli ${ }^{7}$, l'amnistie $^{8}$ ou la réconciliation. D'autre part, face à la notion traditionnelle de pardon : le pardon conditionnel, conditionné par un ensemble d'obligations, de compromis et de buts, et attrapé, par conséquent, dans la logique du calcul, de l'échange, etc., il affirme le pardon inconditionnel - absolument hétérogène et irréductible au pardon conditionnel mais à la fois indissociable de celui-ci : un pardon qui n'a pas à être demandé pour être accordé ; un pardon exceptionnel, infini et pur, désintéressé, gratuit. « Le pardon, s'il y en a, ne doit et ne peut pardonner que l'impardonnable, l'inexpiable - et donc faire l'impossible $»^{9}$, « dans l'endurance infinie de l'im-possible comme possible; et c'est là -ajoute

${ }^{6}$ J. DERRIDA, Pardonner. L'impardonnable et l'imprescriptible. Galilée: Paris, 2012, p. 13.

${ }^{7}$ « C'est le bon sens qui nous rappelle que le pardon n'est pas l'oubli, mais partout où de l'oubli, sous une forme ou sous une autre, par exemple, sous forme de la transformation, de la réconciliation, du travail de deuil, peut s'infiltrer, le pardon n'est plus pur. Le pardon doit supposer une mémoire intégrale en quelque sorte. Je ne crois pas qu'il y ait un devoir de non-pardon, je crois en tout cas à l'hétérogénéité absolue entre le mouvement ou l'expérience du pardon d'une part et tout ce que trop souvent on lui associe, c'est-à-dire la prescription, l'acquittement, l'amnistie, voire l'oubli sous toutes ses formes. L'oubli n'est pas simplement le fait de perdre la représentation du passé, mais simplement de se transformer, de se réconcilier, de reconstituer un autre corps, une autre expérience» (J. DERRIDA, « Justice et pardon », pp. 135-136).

${ }^{8}$ À propos de la Commission Vérité et Réconciliation sud-africaine, Derrida va parler d'une certaine christianisation de l'amnistie de la part de l'évêque anglican Desmond Tutu : " l'amnistie devient pardon, un pardon précédé d'un témoignage accompagné de repentir. Le christianisme subreptice, c'est aussi, au cours de ce devenir-pardon de l'amnistie, l'imposition tentée d'un modéle chrétien du pardon » (J. Derrida, "Versöhnung, ubuntu, pardon: quel genre? », in Le Genre humain, n 43, 2004 [B. CASSIN, O. CAYLA ET PH.-J. SALAZAR, dirs., Vérité, réconciliation, réparation], p. 149-150). Ce texte a été publié plus tard, avec un titre légèrement modifié : « Le pardon, la vérité, la réconciliation : quel genre ?», in J. DERRIDA, La Solidarité des vivants et le pardon, pp. 61-120).

${ }^{9}$ J. DERRIDA, Pardonner. L'impardonnable et l'imprescriptible, p. 28. Voici un autre texte de Derrida : «Dès lors que la possibilité du pardon, s'il y en a, consisterait en une certaine im-possibilité, doit-on en conclure qu'il faut alors faire l'impossible ? Et le faire avec des mots, seulement avec des mots ? Faut-il faire l'impossible pour qu'advienne un pardon comme tel ? / Peut-être, mais on ne peut pas ériger cela en loi, en norme, en règle ou en devoir. Il ne devrait pas y avoir de "il faut" pour un pardon. Celui-ci "doit" toujours rester gratuit et imprévisible. On ne donne ni ne pardonne jamais "conformément à un devoir" (pflichtmässig), pas même "par devoir" (eigentlich aus Pflicht), pour reprendre la bonne distinction kantienne. On pardonne, si l'on pardonne, par-delà tout impératif catégorique, au-delà de la dette et du devoir. Et pourtant il faudrait pardonner. Que suppose en effet le pardon infini, le pardon 
Derrida- ce qu'il aurait en commun avec le don $»^{10}$. Tout comme le don, le pardon « digne de ce nom » doit d'ailleurs ne pas être reconnu en tant que tel, il doit être ignoré en tant que tel, puisqu'il ne peut être que sans calcul, sans dette, sans échange, sans contrepartie, sans réciprocité. Mais, dans la mesure où il est exceptionnel et que, partant, il interrompt le cours de l'histoire ${ }^{11}$, ce pardon inconditionnel, dépourvu de sens, de limite et de finalité ${ }^{12}$, constitue également un évènement : un évènement toujours inattendu, imprévisible, impossible et, par conséquent, à l'épreuve de cette justice indéconstructible, de cette éthique de la responsabilité au-delà des lois, des règles et des devoirs, qu'exige une pensée comme celle de Derrida.

C'est évidemment sur le Chili, son pays, sur le Chili post-Pinochet, qu'Agüero jette, d'autre part, son dévolu afin de fournir au lecteur de son livre un exemple précis et très à propos de ce pardon prétendument politique et juridique qui a pour finalité une réconciliation cherchant à reconstruire l'unité nationale, à rétablir la normalisation sociale, à reconstituer le corps de l'État-nation. Or, la rhétorique du pardon ainsi que ses procédés ne demeurent-ils pas étrangers, comme l'affirme Derrida, à une justice réelle?

Quel rôle a joué le pardon, dans ce « pays d'Antigones », lors de la transition de la dictature de Pinochet à un régime démocratique ? Comment, au nom d'une politique de pardon institutionnalisé et d'une réconciliation nationale, la société chilienne affrontée, déchirée et à tout jamais endeuillée par ces milliers de détenus, de disparus et de " morts sans sépulture » a-t-elle vécu cette transition assurément beaucoup trop longue et souvent taxée de partielle, voire de continuiste, qui ne s'engage à faire justice que « dans la mesure du possible » et qui, dans un premier temps déjà, ne semble pas prête à réprouver vraiment ce que, bon gré mal gré, elle va hériter du régime précédent? Une transition qui, en fait, s'est effectuée par tractation entre des partis qui, dans un passé encore tout récent, étaient nonobstant des adversaires politiques irréconciliables, et dans le cadre de laquelle vont longtemps

\footnotetext{
"hyperbolique", et donc inconditionnel, celui dont le "commandement" semble nous venir, par héritage, de la tradition abrahamique, relayée de façons différentes par saint Paul et par le Coran? Suppose-t-il, comme sa condition (condition de l'inconditionnalité même, donc) que le pardon soit demandé et la faute avouée, comme le rappelle si fortement Jankélévitch ? Mais alors il ne serait plus inconditionnel. De nouveau conditionné, il ne serait plus inconditionnel. De nouveau conditionné, il ne serait plus le pardon pur, il redeviendrait impossible, autrement impossible. Ou bien ne peut-il être inconditionnel, et donc possible comme inconditionnel, qu'à pardonner l'impardonnable (donc à devenir possible comme impossible) ? Ne peut-il être ce qu'il doit être, inconditionnel, qu'à ne même plus exiger cet aveu ou ce repentir, cet échange, cette identification, cet horizon économique de réconciliation, de rédemption et de salut ? » (J. DERRIDA, « Comme si c'était possible, "within such limits"... », in Papier Machine, Galilée: Paris, 2001, p. 294).

${ }^{10}$ J. DERRIDA, Pardonner. L'impardonnable et l'imprescriptible, p. 68.

${ }^{11}$ « Il n'y a pas d'histoire sans pardon, sans réconciliation, etc. et en même temps il y a dans le pardon quelque chose de transcendant à l'histoire, qui l'interrompt, qui se porte au-delà dans un instant paradoxal, incalculable, comme un instant de folie... » (J. DERRIDA, « Justice et pardon », p. 141).

${ }_{12}$ «Un pardon "finalisé" n'est pas un pardon, c'est seulement une stratégie politique ou une économie psychothérapeutique» (J. DERRIDA, « Le siècle et le pardon », p. 124).
} 
cohabiter les institutions -apparemment encore non- révolues de la dictature et les nouvelles institutions d'un État de droit. Une transition qui se déroule partant, pour ainsi dire, sous tutelle - ladite dictature ayant déjà pris à l'avance un certain nombre de dispositions constitutionnelles, politiques et juridiques qui vont lui permettre d'en maîtriser le développement et d'en ralentir le progrès. Tel est, par exemple, le cas du décret-loi d'amnistie de 1978, amnistie faussement générale puisque destinée, en fait, à garantir l'impunité des militaires pour les crimes commis de 1973 à 1978; d'une série de lois -connues sous le nom de " lois d'amarrage »-concernant le système judiciaire; de la loi électorale établissant un système anti-démocratique de scrutin binominal pour les élections parlementaires ; voire de la Constitution même du Chili établie en 1980 par les militaires mais encore amendée à leur profit en 1988 ! Les successifs gouvernements de la transition ont sans doute eu l'occasion, à plusieurs reprises, de remettre en cause, voire de changer ou d'abolir, toutes ces lois. Cependant, le plus souvent ils ont décidé -sans doute au nom de la réconciliation-de les maintenir et de négocier pas à pas certains amendements et certaines réformes démocratiques plus ou moins relevants.

Et Pinochet, ce dictateur qui nonobstant va demeurer, jusqu'en 1998, à la tête des armées pour devenir ensuite sénateur à vie, ce dictateur dont l'immunité constitutionnelle qu'il s'était garantie au préalable et qui n'a été supprimée qu'en 2004, en raison de sa participation à l'opération Condor, a de nouveau été ratifiée par la Cour Suprême en 2005, est-ce que ses victimes l'ont pardonné ? Est-ce qu'elles ont pardonné leurs tortionnaires, leurs bourreaux ? Est-ce que la société chilienne a pu oublier toutes les abominations, toutes les atrocités, qu'elle a dû endurer sous la dictature?

Quant aux victimes, ne les a-t-on pas négligées, délaissées, au nom de la réconciliation nationale, pendant trop longtemps ? Certes, des mesures de réparation économique ont été parfois mises en vigueur à leur égard. Mais, une compensation, un dédommagement de cette nature, répondent-ils, de fait, à la ferme intention de faire amende honorable ou bien s'agit-il là, plutôt, tout simplement d'un certain calcul stratégique et politique cherchant à manipuler ces victimes et/ ou leurs proches en achetant ainsi leur silence ? Qu'en est-il « de la dignité civile et humaine des victimes » $?^{13}$ À la suite surtout de multiples mobilisations sociales, des commissions sont mises en place dans le dessein d'établir et de faire constater qu'il y a bien eu, sous la dictature militaire, un terrorisme d'État qui a laissé sur son passage d'innombrables victimes. Ainsi, la Commission nationale de Vérité et de Réconciliation (Rapport Rettig) (1990-1991) va s'occuper d'informer sur les violations des droits de l'homme commises durant cette période et d'en identifier les victimes ; une « Table de dialogue sur les droits de l'homme » (1999-2001) donne lieu à un rapport sur les disparus ainsi qu'à la reconnaissance, de la part des

${ }^{13}$ J. DERRIDA, « Versöhnung, ubuntu, pardon: quel genre? », pp. 117-118. 
autorités militaires, des multiples violations de ces droits ; la Commission Valech (2004) va enquêter sur les tortures et l'emprisonnement politique. Malgré leur effet salutaire, ces commissions vont toutefois susciter un certain nombre d'objections et de reproches : critères trop étriqués, silence sur les tortionnaires et les bourreaux, absence de procédures et de poursuites judiciaires, etc.

Il ne fait aucun doute que la lutte contre l'impunité a manqué de fermeté et que, tout comme la politique officielle de mémoire historique, elle s'est faite attendre trop longtemps. C'est aussi pourquoi, affirme Agüero, lesdites commissions n'ont constitué, au bout du compte, que des opérations historiques « anti-mémoire ». Autant d'exemples, parmi tant d'autres, de la façon dont la mémoire historique (le devoir de mémoire passée et future) et la recherche de la vérité, "la révélation et l'établissement sans concession de la vérité $\gg^{14}$-pour l'archiver ${ }^{15}$ et la rendre publique-, courent toujours le risque d'être faussées, dénaturées, par cette autre soidisant vérité, cette « vérité officielle » lors de laquelle la mémoire, affirme l'auteur, « est prise en otage par le récit officiel tributaire du grand consensus social que suppose » l'histoire.

Est-ce que la transition chilienne est achevée d'une fois pour toutes ? Est-ce que le Chili post-Pinochet a réussi, malgré tout, à se réconcilier enfin avec lui-même? Est-ce qu'il a oublié ? Est-ce qu'il a pardonné ? Ou bien est-ce que les spectres de Pinochet, de ses suppôts, des tortionnaires, hantent toujours ce Chili post-dictature?

Agüero a raison de déclarer qu'il vaut mieux ne pas conclure. À plus forte raison dès lors que l'on dit " oui, oui », que l'on dit « viens ", à cette justice indéconstructible, impossible et toujours à-venir ; dès lors que l'on affirme cette «éthique au-delà de l'éthique, voilà peut-être -ajoute Derrida- le lieu introuvable du pardon $»^{16}$.

\footnotetext{
${ }^{14}$ J. DERRIDA, «Versöhnung, ubuntu, pardon: quel genre? », p. 147.

${ }^{15}$ « [...] qui disposera de cette archive ? Qui en contrôlera l'accès ? Qui travaillera à l'interprétation de cette histoire, des récits, des témoignages, de leur interprétation même, etc. ? Que le gouvernement luimême [Derrida parle ici de l'Afrique du Sud] ne soit pas disposé à renoncer au contrôle de cette archive peut paraître inquiétant, même s'il peut alléguer à cet égard un souci d'apaisement et, surtout, respecter avec conséquence la décision prise initialement, à savoir d'accorder un temps limité à ce processus de deuil et de pardon » (Ibid. C'est moi, C.P., qui mets les crochets).

${ }^{16}$ J. DERRIDA, « Le siècle et le pardon », p. 111.
} 